\title{
Previsibilidade e eficiência no mercado agrícola
}

\author{
Predictability and efficiency in agricultural market
}

\section{Marcelo Brutti Righi ${ }^{I}$ Paulo Sergio Ceretta ${ }^{I I}$}

\section{RESUMO}

\begin{abstract}
O presente trabalho possui como objetivo testar a hipótese de caminho aleatório no mercado da Soja, Algodão, Café e Milho, através da utilização de testes de quociente de variância. São utilizados dados referentes às cotações diárias dos indicadores de preço da ESALQ/CEPEA, correspondentes ao período de 13/03/2006 a 20/10/2010. Os resultados obtidos permitem concluir que todas as commodities rejeitam a hipótese de caminho aleatório, e por consequência a eficiência de mercado, gerando oportunidade de arbitragem.
\end{abstract}

Palavras-chave: caminho aleatório, quociente de variância, commodities.

\begin{abstract}
This research aims to test the random walk hypothesis in Soybean, Cotton, Coffee and Corn markets by using the variance ratio tests. The data used refers to the daily quotations of price indicators, ESALQ/CEPEA for the period from 13/03/2006 to 20/10/2010.Results allowed to conclude that all commodities reject the random walk hypothesis, and hence the efficiency of market, generating arbitrage opportunity.
\end{abstract}

Key words: random walk, variance ratio, commodities.

\section{INTRODUÇÃO}

A possibilidade de prever o comportamento futuro de preços de ativos tem sido alvo constante de estudos desenvolvidos pelos pesquisadores da área financeira. Este fato é alavancado pela possibilidade de arbitragem, que traria como consequência ganhos anormais. Não obstante, através de diversas metodologias desenvolvidas com o decorrer do tempo, foram identificados ativos com comportamento previsível, em algum grau, em diferentes segmentos da economia.

De acordo com FAMA (1970), ao se falar em previsibilidade do mercado de ações, deve-se ressaltar o random walk, ou caminho aleatório, que trouxe importantes contribuições à literatura empírica, pois remete ao fato de que os retornos futuros são independentes das informações passadas. Dessa forma, a hipótese do caminho aleatório traz implicâncias quanto à possibilidade de se conseguir prever, de alguma maneira, com base em retornos passados, os retornos futuros, tirando proveito disso para auferir rendimentos extraordinários.

Trabalho pioneiro, de acordo com HOQUE et al. (2007), sobre testes de quociente de variâncias foi desenvolvido por LO \& MACKINLAY (1988), referenciado como teste de quociente de variâncias simples. Segundo os autores, tal teste foi criado sob duas suposições, com o intuito de capturar duas facetas do caminho aleatório: (a) inovações independentes e identicamente distribuídas na forma de uma distribuição normal (i.i.d.); e (b) inovações não correlacionadas, mas fracamente dependentes e com a possibilidade de heteroscedasticidade em sua distribuição de frequência (m.d.s.). O ponto crucial desse teste é que, se o retorno

'Departamento de Ciências Administrativas, Universidade Federal de Santa Maria (UFSM), Santa Maria, RS, Brasil.

IPrograma de Pós-graduação em Administração, UFSM, 97105-900, Santa Maria, RS, Brasil. E-mail: ceretta@ufsm.br. Autor para correspondência. 
de uma ação segue um caminho puramente aleatório, a variância do retorno de um período $q$ é $q$ vezes a variância da primeira diferença. Assim, a hipótese nula do teste afirma que a razão das variâncias seja igual a 1 . O teste proposto por LO \& MACKINLAY (1988) é exposto na formulação [1].

$V R\left(y ; k_{i}\right)=\left\{\frac{1}{T k} \sum_{t=k+1}^{T}\left(y_{t}+\cdots+y_{t-k}-k \mu\right)^{2}\right\} \div\left\{\frac{1}{T} \sum_{t=1}^{T}\left(y_{t}-\mu\right)^{2}\right\}$

$\mathrm{Na}$ formulação [1], $y_{\mathrm{t}}$ corresponde à observação de uma série temporal no instante $t$; $\widehat{\mu}=T^{-1} \sum_{i=1}^{T} y_{t} ; k$ é o número de defasagens utilizadas; $T$ é o número de observações da série temporal. Se $y_{\mathrm{t}}$ é i.i.d., então a estatística $M_{\mathbf{1}}\left(y, k_{i}\right)$, representada pela formulação [2], segue assintoticamente a distribuição normal padrão. Entretanto, tal suposição não se mantém quando $y_{\mathrm{t}}$ apresenta heteroscedasticidade condicional (AZAD, 2009). Visando a contornar essa dificuldade, LO \& MACKINLAY (1988) propõem um teste estatístico robusto. Essa estatística $M_{3}\left(y, k_{i}\right)$, que também segue a distribuição normal padrão, devendo ter como critério de rejeição para um teste de hipóteses um valor crítico baseado em determinado nível de significância, está representada matematicamente pela formulação [3].

$$
\begin{aligned}
& M_{\mathbf{1}}\left(y, k_{i}\right)=[V R(y ; k)-1] \times\left[\frac{2(2 k-1)(k-1)}{3 k T}\right]^{-\frac{1}{2}} . \\
& M_{\mathbf{2}}\left(y, k_{i}\right)=[V R(y ; k)-1] \times\left\{\sum_{j=1}^{k-1}\left[\frac{2(k-j)}{k}\right]^{2} \delta_{j}\right\}^{-\frac{1}{2}} \cdot[3]
\end{aligned}
$$

Na formulação [3],

$\delta_{j}=\left\{\sum_{t=j+1}^{T}\left(y_{t}-\hat{\mu}\right)^{2}\left(y_{t-j}-\not{a}\right)^{2}\right\}=\left\{\left[\sum_{t 1}^{T}\left(y_{t}-\not{\mu}\right)\right]^{2}\right\}$.

A dificuldade de interpretação dos testes $M_{1}$ e $M_{2}$ é que os resultados podem ser conflitantes para diversos valores de $k$. Visando a contornar essa questão, CHOW \& DENNING (1993) propuseram o quociente de variâncias múltiplas (multiple variance ratio test). Tal teste implica, segundo seus autores, uma modificação do teste de LO \& MACKINLAY (1988), em que é possível verificar ao mesmo tempo se todos os quocientes de variância são iguais a 1. Este teste, segundo HOQUE et al. (2007) baseia-se na idéia de que a decisão com relação à hipótese nula pode ser baseada no valor máximo absoluto das estatísticas de quociente de variâncias individuais. A estatística de CHOW \& DENNING (1993) é apresentada na formulação [4].

$M V\left(y ; k_{i}\right)=\sqrt{T \max _{1 \leq i \leq m}\left|M\left(y ; k_{i}\right)\right|}$. [4]
Na formulação [4], $M V\left(y ; k_{i}\right)$ representa o teste estatístico de quociente de variância individual para o período $k_{\mathrm{i}}$; $T$ é o número total de observações da série temporal analisada. Tal estatística segue uma distribuição SMM (student maximum modulus) $\operatorname{com} m$ e $T$ (tamanho da amostra) graus de liberdade, devendose rejeitar a hipótese nula se o valor obtido exceder determinado valor crítico.

Posteriormente, WRIGHT (2000) propôs o uso de testes de quociente de variâncias nãoparamétricos baseados nos postos e nos sinais das séries temporais, para testar a hipótese nula de que essas séries seguem um caminho aleatório. O quociente de variâncias com base nos postos baseia-se, segundo CERETTA (2001), nos postos das variações de preço com desenvolvimento matemático muito próximo ao teste de LO \& MACKINLAY (1988). Dessa forma, WRIGHT (2000) propôs os testes de postos $R_{1}$ e $R_{2}$, representados pelas formulações [5] e [6], e o testes de sinal $S_{1}$, exposto na formulação [7], como alternativa aos testes $M_{1}$ e $M_{2}$. Tais estatísticas seguem a distribuição normal, devendo ter a hipótese nula rejeitada quando o valor calculado exceder determinado valor crítico.

$$
\begin{aligned}
& R_{1}\left(k_{i}\right)=\left[\frac{(1 / T k) \Sigma_{t=k+1}^{T}\left(r_{1, t}+\cdots r_{1, t-k}\right)^{2}}{(1 / T) \Sigma_{t=1}^{T} r_{1, t^{2}}}-1\right] \times\left[\frac{2(2 k-1)(k-1)}{3 k T}\right]^{-\frac{1}{2}} \cdot[5] \\
& S_{1}\left(k_{i}\right)=\left[\frac{(1 / T k) \Sigma_{t=k+1}^{T}\left(s_{t}+\cdots+s_{t-k}\right)^{2}}{(1 / T) \Sigma_{t=1}^{T} s_{t}^{2}}-1\right] \times \\
& {\left[\frac{2(2 k-1)(k-1)}{3 k T}\right]^{-\frac{1}{2}}}
\end{aligned}
$$

A diferença crucial entre os testes de quociente de variância baseado em postos $R_{1}$ e $R_{2}$ se dá pelo cálculo das variáveis integrantes do modelo.

$$
\text { Assim, em [5] }[6], r_{1, t}=r\left(y_{t}\right)-\left[\frac{T+\mathbf{1}}{2} / \sqrt{\frac{(T+1)(T-1)}{12}}\right]
$$

e $r_{2, t}=\phi^{-1}\left[r\left(y_{t}\right) /(T+1)\right]$, em que $r\left(y_{\mathrm{t}}\right)$ é o posto de $y_{\mathrm{t}}$

$\phi$ é a função cumulativa da distribuição normal padrão. O teste de quociente de variância baseado nos sinais dos retornos é exibido na formulação [7].

$S_{\mathbf{1}}\left(k_{i}\right)=\left[\frac{(1 / T k) \Sigma_{t=k+1}^{T}\left(S_{\tau}+\cdots+s_{t-k}\right)^{2}}{(1 / T) \sum_{t=1}^{T} s_{\tau}^{2}}-1\right] \times\left[\frac{2(2 k-1)(k-1)}{3 k T}\right]^{-\frac{1}{2}} \cdot[7]$

Na formulação [7], $s_{\mathrm{t}}=u\left(y_{\mathrm{t}}, 0\right)$, em que $u\left(x_{\mathrm{t}}, 0\right)=1$ se $y_{\mathrm{t}}>0$, e 0 caso contrário. Sob a suposição de que $y_{\mathrm{t}}$ é gerado por uma sequência diferenciada martingale, $s_{\mathrm{t}}$ é uma sequência i.i.d. com média zero de variância unitária, que assume valores 1 e-1 com igual probabilidade. 
Corroborando esse raciocínio, BELAIREFRANCH \& CONTRERAS (2004) enfatizam que é possível estender a ideia por trás do teste de CHOW \& DENNING (1993) para os testes de postos e sinais de WRIGHT (2000). Dessa forma, para testar a hipótese nula conjunta de que $V R\left(y ; k_{\mathrm{i}}\right)=1, i=1,2 \ldots m$, contra a alternativa de $V R\left(y ; k_{\mathrm{i}}\right) \neq 1$, para algum $i$. A decisão de rejeitar ou não a hipótese nula é baseada em determinado valor crítico da distribuição SMM com $m$ e $T$ graus de liberdade. Sendo assim, as formulações [8], [9], [10], apresentam os testes conjuntos de quociente de variância de Wright.

$J R_{1}=\max _{1 \leqq i \Xi m}\left|R_{1}\left(k_{i}\right)\right| \cdot[8]$

$I R_{2}=\max _{1 \leq i \leq m}\left|R_{2}\left(k_{i}\right)\right| \cdot[9]$

$J S_{1}=\max _{1 \leqq i \leqq m}\left|S_{1}\left(k_{i}\right)\right| \cdot[10]$

Visando a lidar com problemas como correlação serial e heteroscedasticidade condicional, CHEN \& DEO (2006) propõem um teste de quociente de variância para grandes horizontes de tempo, com o objetivo de testar a hipótese nula conjunta de que $V R$ $\left(y ; k_{\mathrm{i}}\right)=1, i=1,2 \ldots m$, contra a alternativa de $V R\left(y ; k_{\mathrm{i}}\right) \neq 1$, para algum $i$. Assim sendo, conforme o procedimento de CHEN \& DEO (2006), deve-se rejeitar a hipótese nula de caminho aleatório se, de maneira simultânea:

$\sum_{i=1}^{n}\left[\operatorname{VRP}\left(y ; k_{i}\right)-1\right]<0$ e $Q P_{n}>\chi_{n, 2 \alpha}^{2}[11]$.

Na formulação [11],

$\operatorname{VRP}\left(y ; k_{i}\right)=\operatorname{Var}\left(\sum_{i=1}^{k} \varepsilon_{t}\right) / k\left(\operatorname{Var}\left(\varepsilon_{1}\right)\right)$, em que

$\varepsilon_{1}$ é definido como em [1]; $Q P_{n}$ é a forma quadrática das estatísticas $\operatorname{VRP}\left(y ; k_{\mathrm{i}}\right)$, seguindo a distribuição de qui-quadrado $\left(\chi^{2}\right)$.

O presente estudo possui como objetivo fundamental testar a hipótese de caminho aleatório nos mercados à vista das commodities Algodão, Café, Milho e Soja, através da utilização de testes de quociente de variância. Os dados referem-se aos preços de negociação diários, correspondentes ao período de 13/03/2006 a 20/10/2010.

\section{MATERIAL E MÉTODOS}

Com o objetivo de realizar o presente estudo, foi efetuada uma pesquisa descritiva longitudinal. Nesse sentido, pode-se destacar que pesquisas descritivas têm como objetivo primordial a descrição das características de determinada população ou fenômeno, ou o estabelecimento de relações entre as variáveis. Os retornos dos preços agrícolas são calculados através da diferença de seus logaritmos, conforme [12].

$$
r_{\mathrm{t}}=\ln P_{\mathrm{t}}-\ln P_{\mathrm{t}-1 .} \text {. [12] }
$$

Em [12], $r_{\mathrm{t}}$ é o log-retorno no tempo $t ; P_{\mathrm{t}}$ éo preço no dia $t$. Primeiramente, são analisadas as estatísticas descritivas e o gráfico eda-plot. Posteriormente, por meio do teste $Q$ de LJUNG \& BOX (1978), representado pela formulação [13], que testa a hipótese nula de que os dados são aleatórios contra a alternativa de não aleatoriedade deles, procurou-se identificar a presença de correlação serial na série dos retornos.

$Q=n(n+2) \sum_{k=1}^{h} \frac{\hat{\rho}_{k}^{2}}{n-k} \cdot[13]$

Na formulação [13], $n$ é o tamanho da amostra; $\hat{\rho}_{k}^{2}$ é a autocorrelação da amostra na defasagem $k ; h$ é o número de defasagens. A estatística $Q$ de Ljung e Box segue a distribuição de qui-quadrado $\left(x^{2}\right)$. Posteriormente, foram aplicados os teste de quociente de variância de LO \& MACKINLAY (1988), WRIGHT (2000), CHOW \& DENNING(1993), CHEN\& DEO(2006).

\section{RESULTADOS E DISCUSSÃO}

Com base nos preços de negociação diários das commodities estudadas, foram calculados os logretornos. Em seguida, foram calculadas as estatísticas descritivas desses retornos. Os resultados obtidos para essa primeira análise são expostos na tabela 1 .

É possível verificar, com base na tabela 1, que houve certa paridade entre as commodities no que tange à rentabilidade, pois Soja, Algodão e Milho obtiveram retorno médio muito próximo, com o Café um pouco abaixo. Tal resultado se modifica quando é analisada a mediana dos log-retornos, uma vez que a Soja apresenta o maior valor, seguida do Café, Milho e Algodão. No tocante à volatilidade, ou risco, os valores calculados para variância, e desvio padrão por consequência, acusam que o café possui a maior variabilidade dos log-retornos em relação à média, seguido da Soja, Milho e Algodão, que foi o menos arriscado. A questão da normalidade da distribuição de probabilidade dos log-retornos é auferida com base na assimetria, curtose e do teste de Doornik-Hansen de normalidade. Os resultados indicam que, devido à assimetria (negativa para Soja e Café, e positiva para Algodão e Milho) e curtose (Soja e Café leptocurticas, e Algodão e Milho plactocúrticas) os log-retornos das commodities não possuem distribuição normal, comprovado pelo teste de normalidade de DoornikHansen. Tal falta de normalidade dos retornos é exposta 
Tabela 1 - Estatísticas descritivas dos log-retornos da Soja, Algodão, Café e Milho.

\begin{tabular}{|c|c|c|c|c|}
\hline \multirow{2}{*}{ Estatísticas } & \multirow[b]{2}{*}{ Soja } & \multirow[b]{2}{*}{ Algodão } & \multirow[b]{2}{*}{ Café } & \multirow[b]{2}{*}{ Milho } \\
\hline & & & & \\
\hline Observações & 1146 & 1146 & 1146 & 1146 \\
\hline Mínimo & $-6,425$ & $-2,204$ & $-7,922$ & $-3,270$ \\
\hline $1^{\circ}$ Quartil & $-0,607$ & $-0,140$ & $-0,625$ & $-0,388$ \\
\hline Mediana & 0,069 & 0,009 & 0,043 & 0,013 \\
\hline $3^{\circ}$ Quartil & 0,753 & 0,179 & 0,726 & 0,365 \\
\hline Máximo & 4,765 & 2,670 & 5,732 & 6,054 \\
\hline Média & 0,045 & 0,042 & 0,024 & 0,046 \\
\hline Variância & 1,575 & 0,230 & 2,129 & 0,687 \\
\hline Desvio Padrão & 1,255 & 0,480 & 1,459 & 0,829 \\
\hline Assimetria & $-0,281$ & 0,927 & $-0,509$ & 0,866 \\
\hline Curtose & 1,950 & 5,761 & 2,875 & 5,370 \\
\hline Doornik-Hansen (Normalidade) & 107.355 & 306.886 & 162.613 & 294.979 \\
\hline p-valor & 0.000 & 0.000 & 0.000 & 0.000 \\
\hline
\end{tabular}

Fonte: Elaborado pelos autores.

visualmente por meio dos gráficos eda-plot, exibidos na figura 1. Os gráficos eda-plot presentes na figura 1 evidenciam a assimetria e curtose dos log-retornos. Também é pertinente observar a presença de observações atípicas (outliers).

Em seguida, buscando verificar a dependência temporal nas séries estudadas, com o intuito de escolher as defasagens empregadas nos testes de quociente de variância, calculou-se a estatística $Q$ de LJUNG \& BOX (1978) para 2, 4, 6, 8 e 10 dias de negociação passados. Os resultados obtidos com esse teste são apresentados na tabela 2 .

Conforme exposto na tabela 2 , auferiu-se que os valores calculados para os testes $Q$ de LJUNG \& BOX (1978) empregues são significativos ao nível de $1 \%$ para todas as commodities presentes no estudo para 2, 4, 6, 8 e 10 defasagens. Logo, são estes dias de negociação passados que foram utilizados para compor os testes de quociente de variância na presente pesquisa. Após essa análise preliminar, foram

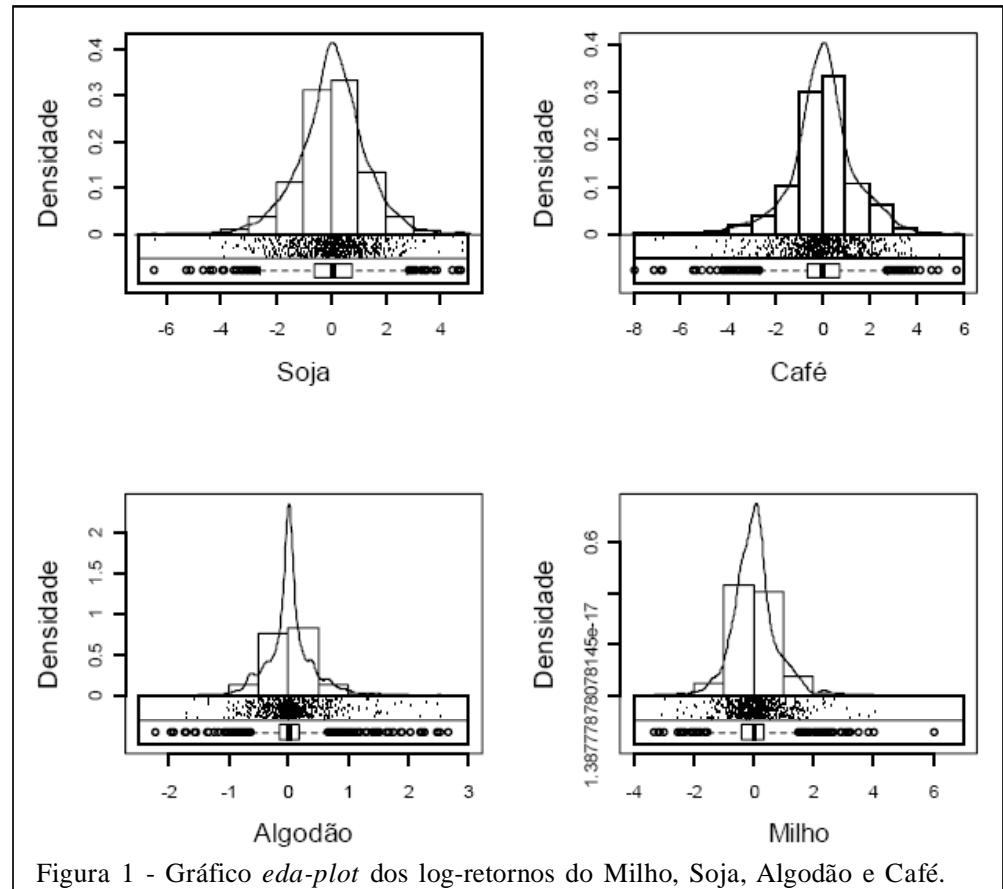

Ciência Rural, v.41, n.10, out, 2011. 
Tabela 2 - Verificação de dependência serial da Soja, Algodão, Café e Milho para 2,4,6,8 e 10 defasagens através da estatística $Q$ de LJUNG \& BOX (1978).

\begin{tabular}{|c|c|c|c|c|c|c|c|c|}
\hline \multirow{2}{*}{ Defasagem } & \multicolumn{2}{|c|}{----------Soja---------- } & \multicolumn{2}{|c|}{--------Algodão-------- } & \multicolumn{2}{|c|}{----------Café---------- } & \multicolumn{2}{|c|}{---------Milho--------- } \\
\hline & Q-stat. & [p-valor] & Q-stat. & [p-valor] & Q-stat. & [p-valor] & Q-stat. & [p-valor] \\
\hline $\mathrm{Q}(2)$ & 14,223 & {$[0,001]$} & 1111,431 & {$[0,000]$} & 20,941 & {$[0,000]$} & 322,261 & {$[0,000]$} \\
\hline $\mathrm{Q}(4)$ & 20,500 & {$[0,000]$} & 1842,362 & {$[0,000]$} & 23,214 & {$[0,000]$} & 579,734 & {$[0,000]$} \\
\hline $\mathrm{Q}(6)$ & 22,358 & {$[0,001]$} & 2248,593 & {$[0,000]$} & 24,917 & {$[0,000]$} & 696,318 & {$[0,000]$} \\
\hline $\mathrm{Q}(8)$ & 22,587 & {$[0,004]$} & 2414,721 & {$[0,000]$} & 25,954 & {$[0,001]$} & 760,601 & {$[0,000]$} \\
\hline $\mathrm{Q}(10)$ & 29,136 & {$[0,001]$} & 2450,338 & {$[0,000]$} & 30,109 & {$[0,001]$} & 785,314 & {$[0,000]$} \\
\hline
\end{tabular}

Fonte: Elaborado pelos autores.

calculados os testes de quociente de variância, começando pelo de LO \& MACKINLAY (1988) e o teste por postos e sinais de WRIGHT (2000). Os resultados estão presentes na tabela 3 .

Os resultados na tabela 3 (Painel A) permitem concluir que, com base nos valores calculados para o teste de LO \& MACKINLAY (1988), todas as commodities rejeitaram a hipótese de caminho aleatório, pois excedem os valores críticos ao nível de $5 \%$ de significância. Por consequência, a hipótese de eficiência de mercado, com exceção da Soja para 6, 8 e 10 defasagens para ambos os testes $M_{1}$ e $M_{2}$ e 4 dias de desfasagen para o teste $M_{2}$. Tais resultados indicam possibilidade de arbitragem para os mercados que rejeitaram a hipótese de eficiência, isto é, possibilidade de realizar lucro sem incorrer em risco.

Tabela 3 - Testes de quociente de variância de LO \& MACKINLAY (1988) e WRIGHT (2000).

\begin{tabular}{|c|c|c|c|c|c|c|}
\hline \multirow{2}{*}{ Commodities } & \multirow{2}{*}{ Estatísticas } & \multicolumn{5}{|c|}{-Defasagen } \\
\hline & & $\mathrm{k}=2$ & $\mathrm{k}=4$ & $\mathrm{k}=6$ & $\mathrm{k}=8$ & $\mathrm{k}=10$ \\
\hline \multirow{3}{*}{ Soja } & 1este & he de va & LO & NAA I (I) & & 05 \\
\hline & M1 & 3,733 & 2,347 & 0,942 & 0,338 & 0,205 \\
\hline & M2 & 2,912 & 1,893 & 0,759 & 0,273 & 0,166 \\
\hline \multirow[t]{2}{*}{ Algodão } & M1 & 24,759 & 37,123 & 43,789 & 47,461 & 49,050 \\
\hline & M2 & 9,993 & 15,271 & 18,386 & 20,369 & 21,518 \\
\hline \multirow[t]{2}{*}{ Café } & M1 & $-3,948$ & $-4,501$ & $-4,271$ & $-3,879$ & $-3,655$ \\
\hline & M2 & $-3,384$ & $-3,768$ & $-3,628$ & $-3,348$ & $-3,184$ \\
\hline \multirow[t]{2}{*}{ Milho } & M1 & 12,975 & 19,638 & 23,770 & 25,688 & 26,671 \\
\hline & M2 & 7,937 & 12,604 & 15,835 & 17,654 & 18,843 \\
\hline & Testes de qu & de variâr & ostos e si & WRIGHT & --------- & 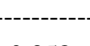 \\
\hline \multirow[t]{3}{*}{ Soja } & $\mathrm{R} 1$ & 2,716 & 1,530 & 0,882 & 0,522 & 0,358 \\
\hline & $\mathrm{R} 2$ & 3,231 & 1,890 & 0,773 & 0,264 & 0,124 \\
\hline & S1 & 1,950 & 0,868 & 0,773 & 0,734 & 0,735 \\
\hline \multirow[t]{3}{*}{ Algodão } & $\mathrm{R} 1$ & 24,696 & 37,709 & 44,674 & 48,871 & 51,203 \\
\hline & $\mathrm{R} 2$ & 24,894 & 37,791 & 44,766 & 48,870 & 50,985 \\
\hline & S1 & 20,028 & 30,616 & 35,976 & 39,351 & 41,438 \\
\hline \multirow[t]{3}{*}{ Café } & $\mathrm{R} 1$ & 5,575 & 5,333 & 4,516 & 3,856 & 3,500 \\
\hline & $\mathrm{R} 2$ & 4,929 & 5,021 & 4,445 & 3,903 & 3,621 \\
\hline & S1 & 3,840 & 3,347 & 2,167 & 1,438 & 1,039 \\
\hline \multirow[t]{3}{*}{ Milho } & $\mathrm{R} 1$ & 13,378 & 21,541 & 26,590 & 29,604 & 31,485 \\
\hline & $\mathrm{R} 2$ & 13,517 & 21,350 & 26,265 & 28,997 & 30,633 \\
\hline & S1 & 10,812 & 16,848 & 20,362 & 22,464 & 23,717 \\
\hline
\end{tabular}

* Resultados significativos ao nível de $5 \%$ em negrito.

Fonte: Elaborado pelos autores. 
Analisando os resultados da tabela 3 (Painel B), verifica-se que o Algodão, o Café e o Milho rejeitaram a hipótese de caminho aleatório para todos os testes e defasagens propostas, pois os valores calculados excederam os valores críticos ao nível de 5\% para todas as estatísticas propostas. A soja rejeitou a hipótese de eficiência de mercado para todos os testes com 2 diasde defasagem e para o teste $R_{2}$ com 4 dias de defasagem. Tais resultados indicam que existe a possibilidade ganhos sem risco nestes mercados, conforme os dias de defasagem citados, pois os preços de negociação destes mercados não refletem totalmente as informações passadas. Os resultados dos testes de quociente de variância conjunta, proposto por $\mathrm{CHOW}$ \& DENNING (1993), CHEN \& DEO (2006) e WRIGHT (2000) estão na tabela 4 (Painel A, Painel B e Painel C).

Os resultados apresentados na tabela 4 (Painel A) evidenciam que as séries compostas pelos log-retornos de todas as commodities rejeitaram a hipótese de caminho aleatório durante o período analisado, pois os valores calculados excedem os pontos críticos ao nível de 5\% para os testes conjuntos. Tal resultado implica a não reflexão imediata das informações passadas nos preços de negociação de Soja, Algodão, Café e Milho, incorrendo na possibilidade de arbitragem. ATabela 4 (Painel B) expõe os valores calculados para o teste de CHEN \& DEO (2006), que é robusto para as questões de heteroscedasticidade e correlação serial. Assim como verificado com o teste de CHOW \& DENNING (1993), todas as commodities rejeitaram a hipótese de caminho aleatório, evidenciando a rejeição da hipótese de eficiência de mercado. Tal resultado implica possibilidade de previsibilidade sobre o comportamento futuro.

Na tabela 4 (Painel C), estão os valores para os testes de quociente de variância de postos e sinais conjuntos, propostos por WRIGHT (2000). Os resultados obtidos indicam que, com exceção do teste $J_{S_{1}}$ para a Soja, todas as estatísticas calculadas obtiveram significância ao nível de 5\%, pois excederam o valor crítico para este nível, rejeitando assim a hipótese de caminho aleatório. Tal resultado tem como consequência a rejeição da hipótese de eficiência de mercado.

Esses resultados podem ser explicados, em parte, por situações como falta de liquidez, assimetria de informação e racionalidade limitada. Mais precisamente, no que tange ao mercado à vista dos ativos presentes nesta pesquisa, com exceção da Soja, o baixo nível de negociações, tanto em concentração, como volume financeiro, pode ser caracterizado como responsável principal pelos resultados encontrados.

\section{CONCLUSÃO}

Conclui-se que todas as commodities rejeitaram a hipótese de caminho aleatório tanto nos testes que consideram defasagens individualmente quanto nas estatísticas conjuntas. Esse resultado implica a rejeição da hipótese de eficiência de mercado na forma fraca. Tal constatação evidencia o fato de que estes mercados não refletem em seus preços todas as informações passadas de forma imediata, o que torna possível certo grau de previsibilidade, ou seja, possibilidade de efetuar lucros sem incorrer em riscos.

Tabela 4 - Resultados para os testes quociente de variância conjunto.

\begin{tabular}{|c|c|c|c|c|}
\hline \multirow{2}{*}{ Estatísticas } & & \multirow[b]{2}{*}{ Café } & \multirow[b]{2}{*}{ Milho } \\
\hline & Soja & Algodão & & \\
\hline CD1 & $\begin{array}{c}\text { nel A - T } \\
3,733\end{array}$ & $\begin{array}{c}\text { DENNIN } \\
49,050\end{array}$ & 4,501 & 26,671 \\
\hline VR(-1) & 0,079 & 1,433 & $-0,296$ & 0,965 \\
\hline QPn & 12,952 & 98,489 & 18,960 & 113,164 \\
\hline JR1 & 2,716 & 51,203 & 5,575 & 31,485 \\
\hline JR2 & 3,231 & 50,985 & 5,021 & 30,633 \\
\hline JS1 & 1,950 & 41,438 & 3,840 & 23,717 \\
\hline
\end{tabular}

* Resultados significativos ao nível de 5\% em negrito.

Fonte: Elaborado pelos autores. 


\section{REFERÊNCIAS}

AZAD, A. S. M. S. Random walk and efficiency tests in the Asia-Pacific foreign exchange markets: evidence from the postAsian currency crisis data. Research in International Business and Finance, 23, 322-338, 2009. Disponível em: $<\mathrm{http}$ ://www.sciencedirect.com/science/article/pii/ S0275531908000548>. Acesso em: 20/10/2010. doi:10.1016/ j.ribaf.2008.11.001.

BELAIRE-FRANCH G.; CONTRERAS D. Ranks and signsbased multiple variance ratio tests.Working paper. Espanha/University of Valencia, 25f. 2004.

CERETTA, P. S. Hipótese do Caminho Aleatório nos mercados da América Latina: aplicação do teste de cociente de variância. 114 folhas. Tese (Doutorado em Engenharia de Produção) - Programa de Pós-Graduação em Engenharia de Produção e Sistemas, Universidade Federal de Santa Catarina, Florianópolis, 2001.

CHEN, W. W.; DEO, R. S. The Variance Ratio Statistic at Large Horizons. Econometric Theory, 22, 206-234, 2006. Disponível em: <http://www.foreignpolicybulletinmonitor.com/
action/displayJournal?jid=ECT $>$. Acesso em: 15/05/2010. doi: $10.1017 / \mathrm{S} 0266466606060099$.

CHOW, K. V.; DENNING, K. C. A simple Multiple Variance Ratio Test. Journal of Econometrics, v. 58, p. 385-401, 1993.

FAMA, E. F. Efficient capital markets: a review of a theory and empirical work. Journal of Finance, vol. 25, p.383-417,1970.

HOQUE, H. A. A. B et al. A comparison of variance ratio tests of random walk: a case of asian emerging stock markets. International Review of Economics and Finance, v. 16, n. 4 p.488-502, 2007. Disponível em: <http://www.sciencedirect.com/ science/article/pii/S1059056006000220>. Acesso em: 20/06/2010. doi:10.1016/j.iref.2006.01.001.

LJUNG, G. M.; BOX, G. E. P. On a Measure of a Lack of Fit in Time Series Models. Biometrika, n.65, pp. 297-303, 1978.

LO, A.W.; MACKINLAY, A.C. Stock market prices do not follow random walks: evidence from a simple specification test. Review of Financial Studies, v.1, p.41-66, 1988.

WRIGHT, J. H. Alternative Variance-Ratio Tests Using Rank and Signs. Journal of Business and Economic Statistics, v. 18 , p.1-9, 2000. 